ORIGINAL ARTICLE

\title{
"Here's egg in your eye": a prospective study of blunt ocular trauma resulting from thrown eggs
}

\author{
R M K Stewart, J M Durnian, M C Briggs
}

Emerg Med J 2006;23:756-758. doi: 10.1136/emj.2006.035501

See end of article for authors' affiliations

.....................

Correspondence to: J M Durnian, St Paul's Eye Unit, Royal Liverpool

University Hospital, Prescot Street, Liverpool L7 9XP, UK; jon_durnian@hotmail. com

Accepted for publication 12 May 2006

\begin{abstract}
Objective: To see if a public awareness campaign might be justified around Halloween with regard to the dangers of egg throwing.

Method: A prospective study was carried out of all patients who attended the St Paul's Eye Unit's Primary Care Department with ocular injuries resulting from a thrown egg over a 14-month period from November 2004. All injuries were classified as minor, intermediate or major and patients were followed up until discharge.

Results: 13 ocular injuries that were attributed to assault with a raw egg were reported. In all the 13 cases, the eggs had been thrown by strangers. 12 of the patients were men and the average age of the victims was 27.9 years. 9 patients were injured in the left eye and there were no bilateral injuries. On presentation, only 1 patient had a visual acuity of $6 / 6,7$ presented at $6 / 9$, with the remainder having $6 / 18$ or worse. All the patients had closed globe injuries. 8 injuries were classified as major injuries. 4 patients had permanent sequelae, with one suffering permanent, severe visual loss.

Conclusions: Although most of our patients showed improvement in visual acuity, there were severe injuries, with the potential for severe ocular morbidity. We conclude that there is sufficient injury caused by this prank to warrant a public health message. At the least this practice should not be promoted by the press.
\end{abstract}

A s anyone who has woken up on 1 November each year and looked out at the chaos left in the wake of the previous night's activities can attest, Halloween and socalled "mischief night" is now becoming an annual excuse for anti-social behaviour ranging from mild annoyance to the sheer dangerous. One particular prank that seems to be becoming more common is the practice of throwing raw eggs either at buildings or at innocent passers-by. Aside from the drycleaning bills, a raw egg can lead to severe ocular injury due to its weight and size, as has been reported previously. ${ }^{12}$ Guy Fawkes night attracts numerous public service announcements, but the hazards of egg throwing, on and around Halloween, seem to go unnoticed.

We carried out a prospective study of all ocular injuries seen in our casualty service that were caused by a thrown egg during a 14-month period to see if a public awareness campaign may be justified, in particular around 31 October.

\section{METHODS}

This was a prospective, observational study of all patients who attended St Paul's Primary Care Department, Royal Liverpool University Hospital, Liverpool, UK, between 1 November 2004 and 31 December 2005, with ocular trauma as a direct result of a thrown egg. All patients were followed up until discharged from care. The patients were treated by the duty ophthalmologist as he or she thought appropriate for the condition. All patients had full anterior segment and dilated posterior segment examination. Description of injuries followed the Birmingham classification ${ }^{3}$ and was graded as minor-lid haematoma, subconjunctival haemorrhage or corneal abrasion; intermediate-transient rise in intraocular pressure (IOP) or trauma uveitis; or major-hyphaema, commotio retinae, retinal detachment, globe breach or longlasting sequelae. All statistics used are purely descriptive.

\section{BLUNT OCULAR TRAUMA}

Blunt trauma to the eye is a common presentation to ophthalmology emergency services and, although on presentation it may not look as serious as penetrating injuries, major morbidity is common. It is essential that a complete ophthalmological examination is carried out, as an eye with minimal (or no) anterior segment damage may have a severe posterior segment injury. Although no consensus is available on the timing of the ophthalmic examination, within $24 \mathrm{~h}$ is adequate if there is no suspicion of globe rupture. The serious sequelae of blunt trauma include

1. Angle recession (posterior contusion deformity of the anterior chamber angle), which is the posterior displacement of the iris root and associated tearing of part of the ciliary muscle. Glaucoma develops in 7-9\% of eyes with angle recession.

2. Vitreous haemorrhage or haemorrhage into the anterior chamber (hyphaema).

3. Commotio retinae-damage to the outer retinal layers due to the force of the injury traversing the eye. On examination by ophthalmoscopy, it appears as a white sheen on the retina and can be associated with retinal tear development.

4. Retinal tear or dialysis and subsequent retinal detachment.

5. Rupture of the choroid or sclera.

\section{RESULTS}

During the study period there were 18651 admissions to our primary care department, and of these 13 were due to ocular injuries attributed to assault with a raw egg (0.07\%; table 1$)$. All 13 eye injuries were due to eggs thrown by strangers.

Twelve patients were men and the average age of the victims was 27.9 years. Nine patients were injured in the left eye and there were no bilateral injuries. On presentation only one patient had a visual acuity of $6 / 6$, seven presented at $6 / 9$

Abbreviation: IOP, intraocular pressure 
Table 1 Classification of ocular injuries in 13 patients

\begin{tabular}{|c|c|c|c|c|c|c|c|c|}
\hline Case & Age & Month & Sex & Eye & Presenting VA & Injury classification & Discharge VA & Sequelae \\
\hline 1 & 27 & November & Male & Left & $2 / 60$ & Major & $3 / 60$ & $\begin{array}{l}\text { Macula damage, angle } \\
\text { recession }\end{array}$ \\
\hline 2 & 17 & April & Male & Right & $6 / 24$ & Major & $6 / 6$ & None \\
\hline 3 & 20 & April & Male & Left & $6 / 18$ & Major & $6 / 5$ & Corneal scar \\
\hline 4 & 21 & May & Male & Left & $6 / 9$ & Major & $6 / 6$ & Retinal detachment \\
\hline 5 & 24 & May & Male & Right & $6 / 9$ & Major & $6 / 9$ & None \\
\hline 6 & 32 & June & Male & Left & $6 / 9$ & Minor & $6 / 9$ & None \\
\hline 7 & 44 & June & Male & Left & $6 / 24$ & Major & $6 / 9$ & None \\
\hline 8 & 22 & July & Male & Right & $6 / 18$ & Major & $6 / 9$ & Angle recession \\
\hline 9 & 16 & October & Male & Left & $6 / 9$ & Major & $6 / 6$ & None \\
\hline 10 & 20 & October & Female & Right & $6 / 9$ & Minor & $6 / 9$ & None \\
\hline 11 & 50 & October & Male & Left & $6 / 9$ & Minor & $6 / 9$ & None \\
\hline 12 & 54 & October & Male & Left & $6 / 6$ & Intermediate & $6 / 5$ & None \\
\hline 13 & 16 & October & Male & Left & 6/60 (amblyopic) & Intermediate & $6 / 60$ & None \\
\hline
\end{tabular}

with the remainder having $6 / 18$ or worse (one had a visual acuity of 6/60 but was densely amblyopic).

All patients had closed globe injuries; however, only three injuries were classed as minor with corneal abrasions, subconjunctival haemorrhage or, as in one patient, a simple lid haematoma. As would be expected with such injuries, all were given antibiotic treatment and discharged from care.

Two injuries were classed as intermediate injuries. One patient presented with an amblyopic eye with a corneal abrasion and traumatic uveitis - this had settled at the 1week review after treatment with steroid and antibiotic. On presentation, the vision was $6 / 60$ due to amblyopia and on discharge this remained unchanged. The second case was a subconjunctival haemorrhage with traumatic uveitis that again settled after appropriate treatment.

Most of the injuries $(n=8)$ were classified as major ocular injuries. Five of these had various combinations of commotio retinae, IOP rise and hyphaema, all of which settled after appropriate treatment. We will discuss the remaining three cases in detail.

Case 1 was a 27-year-old man who presented with markedly reduced visual acuity (2/60) after being hit with an egg while he was a passenger in a moving car. Examination showed subconjunctival haemorrhage and corneal abrasions; however, there was marked commotio of the macula region (Berlin's disease; fig 1). On review, although the anterior segment injuries and, clinically, the commotio settled, the patient's vision did not improve. Electrodiagnostic testing 2 months after the incident showed permanent damage to the middle and outer retinal layers of the macula corresponding to the photoreceptor layer. Testing showed the ganglion cell layer to be functioning. Clinically, he developed mottling of the macula region, signifying retinal pigment epithelium damage. In addition, there was angle recession of the anterior segment, giving the patient a lifelong risk of developing glaucoma. On discharge, his vision remained poor at $3 / 60$.

Case 4 presented, immediately after an assault with an egg thrown from a passing car, with pain and mildly reduced vision. Examination showed a subconjunctival haemorrhage, hyphaema, mild vitreous haemorrhage and extensive commotio retinae involving the macula; no retinal breaks were identified. Over the next few visits he gradually improved. However, 35 days after the injury he sneezed and noticed an immediate drop in visual acuity (6/18). Examination showed a marked vitreous haemorrhage, and due to the mechanism of injury he underwent vitrectomy and cryotherapy to a large inferior retinal tear and gas endotamponnade. Follow-up in our vitreoretinal service showed satisfactory progress, but 3 months after the initial injury he developed a macula on retinal detachment that necessitated further vitrectomy with

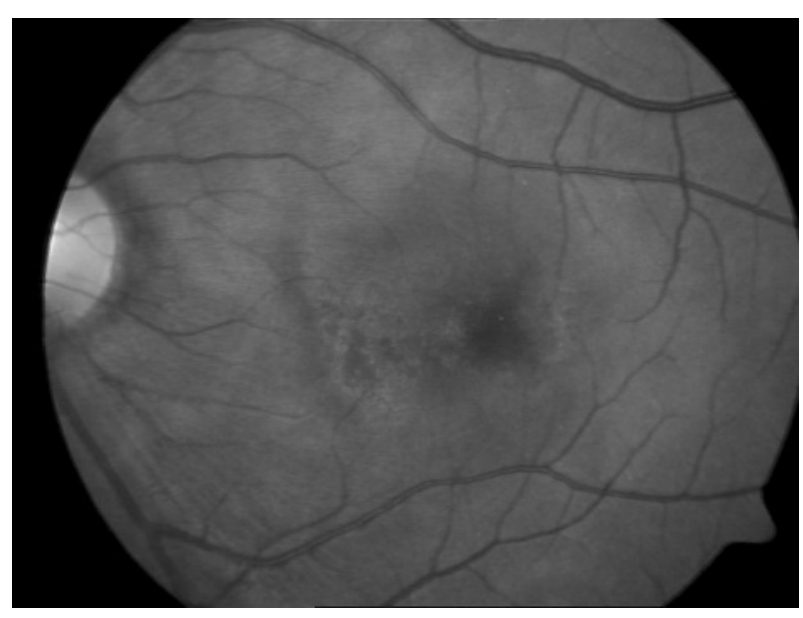

Figure 1 Marked commotio of the macula (Berlin's disease).

gas tamponnade. This treatment was successful and the patient was discharged from care with no retinal detachment and vision of 6/6, six months after the incident.

Case 8 was a 22 -year-old man who presented immediately after the alleged assault with a visual acuity of 6/18. On examination, there was a large corneal abrasion, small hyphaema with secondary rise in IOP $(31 \mathrm{~mm} \mathrm{Hg}$ ), and extensive inferior commotio retinae with some peripheral retinal haemorrhages. As the hyphaema settled, inferior angle recession was also noted. On day 34 after the injury, his vision had improved to $6 / 9$ and IOP was normal, with no drugs being taken. A thorough examination showed no retinal breaks. As with case 1, this patient now has a higher probability of developing glaucoma throughout his life and must undergo yearly checks with his ophthalmologist.

\section{COMMENTS}

Ocular trauma of any sort accounts for a considerable proportion of cases seen in ophthalmic primary care clinics-38-52\% according to previous studies. In 1996, Desai et $a l^{4}$ reported on all ocular trauma cases admitted to Scottish hospitals, and assault accounted for $21.8 \%$ of these cases. Our case series shows similarity to these results in that most of the victims are young men. There was no mention of assault with thrown egg in the 1996 paper, and that would seem coincident with the rise in popularity of this prank. There have been previous reports of ocular trauma caused by thrown eggs elsewhere. In 1988, around Halloween, a series of five cases was reported in the US and two of these patients 
had permanent loss of vision. ${ }^{2}$ In 2003, three patients were reported from Ireland, one of whom had severe visual loss. ${ }^{1}$

Thankfully, with one exception, all our patients did recover well, although when looking at the major injuries in detail, we find some potentially serious injuries such as macula damage, retinal detachment and angle recession. Although the final visual acuities in most cases are reassuring, we must look at the potential serious long-term damage when discussing such injuries. One limitation of our study is that our department does not deal with childhood injuries and this may have led to under-reporting. However, we may expect the younger members of our community to do much of the egg throwing, but their targets may be the older population.

The dimensions of an egg are similar to those of a squash ball, with a considerably greater weight, meaning that eggs, as missiles, can easily fit in the orbital rim, causing severe blunt injury even when thrown by hand. In our series, there were no cases of open globe injury, which is the immediate fear with such a mechanism of injury, but other sequelae of severe, blunt trauma-hyphaema, commotio, retinal tears, haemorrhage-were present.

As is evident from the table, although these cases are spread across the period, there is a cluster in October, which is coincident with the Halloween season. This sort of mischief can be interpreted as innocent, but as seen in our series, can lead to severe ocular morbidity. For years now, resources have been placed on public education surrounding the dangers of fireworks, but no mention has been made of the dangers of egg throwing either around Halloween or at other times. Obviously, you cannot educate people against throwing objects at each other; you rely on their common sense. However, the recent advertising stunt by a leading supermarket in re-branding their eggs as mischief eggs (fig 2) must at least be considered to be irresponsible and at worst almost incitement to this type of assault. The medical community should expect those with most access to the nation's conscience-advertisers, retailers and TV programme makers - to act in a responsible manner against these and other easily preventable injuries.

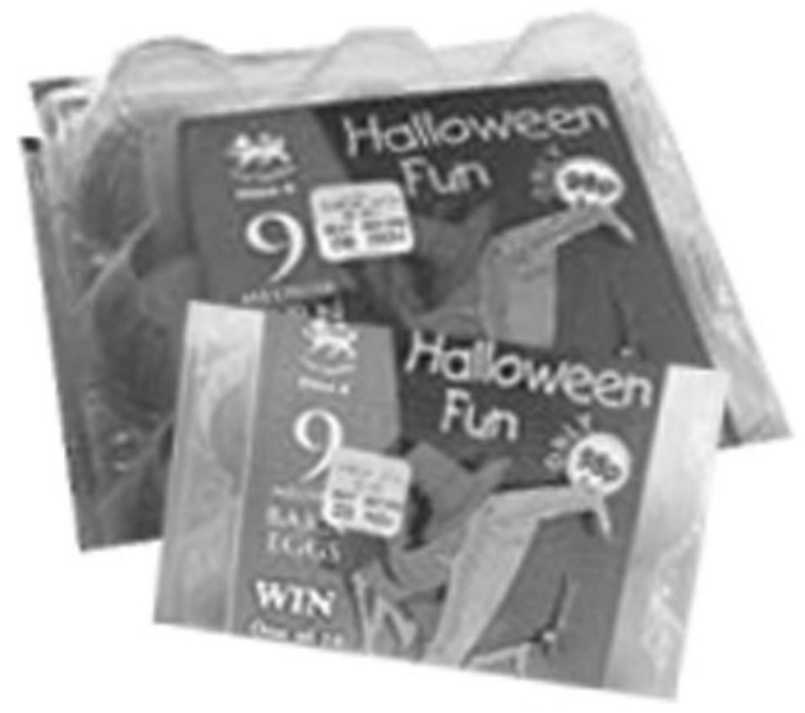

Figure 2 A commercial brand of eggs.

\section{Authors' affiliations}

R M K Stewart, J M Durnian, M C Briggs, St Paul's Eye Unit, Royal Liverpool University Hospital, Liverpool, UK

Competing interests: None.

\section{REFERENCES}

1 O'Toole L, Long V, O'Keefe M, et al. The ocular hazards of egg throwing. Eye 2003;17:278-9.

2 Fiore $\mathbf{P}$, Wagner R. Halloween hazards: ocular injury from flying eggs. N Engl J Med 1988;319:1159.

3 Kuhn F, Morris R, Witherspoon C, et al. A standardized classification of ocular trauma. Graefe's Arch Clin Exp Ophthalmol 1996;234:399-403.

4 Desai P, MacEwen C, Baines P, et al. Epidemiology and implications of ocular trauma admitted to hospital in Scotland. J Epidemiol Commun Health 1996;50:436-41. 